\title{
Tribologists on the Path of Tactile Perception
}

\author{
Francesco Massi ${ }^{1}$ \\ ${ }^{1}$ Department of Mechanical and Aerospace Engineering - Sapienza University of Rome, Italy, francesco.massi@uniroma1.it
}

\begin{abstract}
Between human senses, tactile perception is nowadays object of several research area, due to the lack of knowledge and to its crucial role on several social and industrial issues. Being the contact between skin and touched surfaces at the origin of the tactile sense, tribologists play a key role in the identification and understanding of the signals at the origin of touch. In particular, the quasi-static deformation of the fingertip skin, due to distributed contact forces, together with its transient deformation, due to the vibrations induced by the sliding of the fingerprints on the surface, activate the mechanoreceptors, who collect information to the brain. In this work, the main contributions dealing with the role of friction-induced vibrations and skin-surface contact on tactile perception are discussed.

Keywords-Tactile perception, friction-induced vibrations, skin, biotribology.
\end{abstract}

\section{INTRODUCTION}

Tribology is all around the daily life of human beings. The simplest actions, like walking or grasping a glass of water, are strictly related to the correct tribological response of the interfaces. At the same time, human joints, as hip joints or knees, are without any doubt the most advanced ones, which human technology tries to mimic as close as possible.

Concerning human senses, tactile perception is consequence of a direct contact interaction between the skin and the touched surface. Even if touch is one of the main source of information for sensing the world around us, it is probably the most unknown and still not understood way of perception. The signals at the origin of hearing, acoustic waves, are well known, they can be recorded and reproduced by acoustic speakers; similarly, electromagnetic waves, at the origin of sight, can be recorded and reproduced by a screen. The features of these signals, like tone or amplitude, are directly related to perceived features like color, notes, etc.

On the other side, few is known about the signals at the origin of tactile perception. There is not even a clear understanding in which signals are at the origin of the touch and even less information about the different properties of such signals, which could be associated to respective tactile descriptors, e.g. rough, smooth, sticking, etc.

The understanding of touch is made even more complex by its own nature, which is a combination of several submodalities: temperature and pain, discriminative touch and the kinesthetic sense.

Different sensory systems and tactile afferents are dedicated to the transduction of the different sensory information: thermoreceptive units, nociceptive units and mechanoreceptive units. In particular, the mechanoreceptors, responsible for discriminative touch, are the most densely

Digital Object Identifier (DOI):

http://dx.doi.org/10.18687/LACCEI2019.1.1.480

ISBN: 978-0-9993443-6-1 ISSN: 2414-6390 distributed on the hands and in particular on the fingertip [1].

The mechanoreceptors are the tactile afferents that translate the mechanical stimuli within the skin in electrical signals to be sent to the brain. They are distinguished mainly in Pacinian corpuscles, Ruffini endings, Meissner corpuscles and Merkel disks [2]. As a function of the time response (frequency range) and spatial resolution, they can be classified as slow adapting receptors (SA) and rapidly adapting receptors (RA), with small (Type I) and large (Type II) receptive field. Table I presents the different afferent units, their main function and their characteristic frequency range.

TABLE I

MECHANORECEPTOR TYPES

\begin{tabular}{|l|c|c|}
\hline \multicolumn{1}{|c|}{ Type } & Main features & $\begin{array}{c}\text { Frequency } \\
\text { range }\end{array}$ \\
\hline \hline $\begin{array}{l}\text { Pacinian corpuscles } \\
\text { (RA II) }\end{array}$ & $\begin{array}{l}\text { large receptive field and very } \\
\text { poor spatial resolutions, high } \\
\text { frequency sensitivity }\end{array}$ & $40-500 \mathrm{~Hz}$ \\
\hline $\begin{array}{l}\text { Ruffini endings } \\
\text { (SA II) }\end{array}$ & $\begin{array}{c}\text { poor spatial resolution, } \\
\text { sensitivity to lateral stretching } \\
\text { and high frequency vibrations }\end{array}$ & $100-500 \mathrm{~Hz}$ \\
\hline $\begin{array}{l}\text { Meissner corpuscles } \\
\text { (RA I) }\end{array}$ & $\begin{array}{l}\text { discriminate very fine spatial } \\
\text { details, low frequency vibrations } \\
\text { and tangential deformation }\end{array}$ & $3-40 \mathrm{~Hz}$ \\
\hline $\begin{array}{l}\text { Merkel disks } \\
\text { (SA I) }\end{array}$ & $\begin{array}{c}\text { discriminate spatial details, } \\
\text { such as points, edges, corners, } \\
\text { and curvature }\end{array}$ & $2-16 \mathrm{~Hz}$ \\
\hline
\end{tabular}

As shown above, the afferent units dedicated to the discriminative touch are activated by mechanical stimuli, i.e. the spatial distribution and the transient variation of the stressstrain state within the skin. It is then clear that the "signals" at origin of the tactile perception are the contact forces (normal and frictional stresses along the fingertip surface) and the vibrations induced by the scanning of a surface with the finger.

The roles of induced vibrations and contact forces were already investigated by David Katz [3], who stated the duplex theory of tactile texture perception. He discussed how, while coarse textures are discriminated by static touch (spatial distribution of the skin deformation), fine textures can be discriminated only by introducing a relative motion between fingertip and touched surfaces. This motion is at the origin of friction-induced vibrations, which are a main topic in tribology and are here investigated within their role in tactile perception. Nevertheless, the tribological analysis of the mechanical "signals" at the origin of tactile sense have to be discussed within a more interdisciplinary context, as in the framework of the research networking GdR TACT [4], which combines efforts from researchers on different disciplines: from tribologists to psychologists, from microbiologists to neurologists. 


\section{FRICTION-INDUCED VIBRATION AND TOUCH}

\section{A. Reproduction and analysis of tactile signals}

When dealing with tactile perception, the related tribological signals are extremely complex to be recorded and analyzed. The stimuli are due to the local deformation of the skin, as a consequence of the local interactions between fingerprints and surface reliefs, and the corresponding vibrations induced by such interaction. As in all tribological experiments, a local measurements of forces and deformations at the contact is not possible without modifying the contact itself. For this reason, the only way to recover information is performing vibrational or force measurements as close as possible to the contact. Moreover, when dealing with touch, the vibrational signals are extremely low in amplitude, needing specific test benches, able to control the contact boundary conditions without introducing parasite noise. Fig. 1 presents a tribometer designed to reproduce and measure friction-induced vibrations and contact forces during the scanning of a surface with a finger [5]. The vibrational signals are measured by an accelerometer on the finger nail and are mediated by the transfer function of the fingertip. The vibrational and force signals are then analyzed and their amplitudes and spectral distributions are correlated with the surface features of the scanned samples. The analyses allowed for identifying the frequency content of the signals as a discrimination factor between surface textures [6], in agreement with the duplexperception theory obtained previously by neurophysiological studies [3]. Direct correlation between force and vibrational signals were identified when scanning periodic, isotropic or textured (textile) surfaces [7].

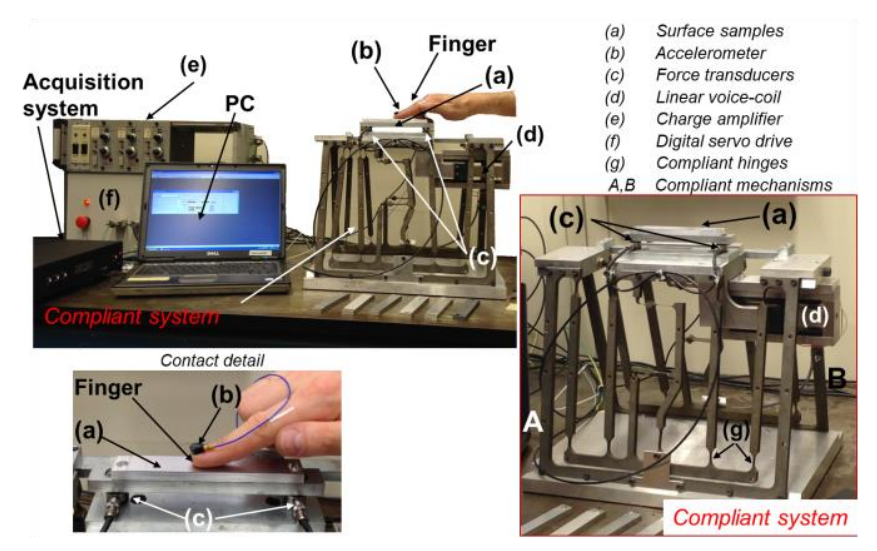

Fig. 1 Example of test bench for reproducing and analyzing tactile signals.

\section{B. From mechanical stimuli to perception}

Even if a correlation between tribological signals and surface properties can be established, the perception passes through cognitive and perceptive mechanisms, which are own of the human brain and have a certain degree of subjectivity. This is why the signals have to be analyzed with respect to the whole cognitive process, from the brain activation due to the signals [8], up to the psychological response of panels of subjects who touch the same surface samples [9]. Topographical surface features, vibrational signals and descriptors from perceptive panels have been compared. These works allowed to highlight how the tactile perception of surfaces is not directly related to the surface topographical properties, but are mediated by the forces and vibrations induced by the contact with the skin [10], which are the signals responsible for the activation of the tactile afferents. Moreover, objective indexes from vibrational signals have been associated with the categorization of the tested samples with respect to specific tactile descriptors [10].

\section{CONCLUDING REMARKS}

The complexity of the cognitive process at the basis of tactile perception, together with the difficulties in reproducing, measuring and analyzing the signals at the origin of touch, make the understanding of tactile perception still a challenge for the scientific community. During the last decades meaningful information have been retrieved on the signals at the origin of touch, but still strong efforts have to be produced in order to identify the objective features of these signals, which are at the origin of our feelings of the tactile interaction with surrounding world.

\section{REFERENCES}

[1] Johansson RS, Vallbo AB. "Tactile sensibility in the human hand: relative and absolute densities of four types of mechanoreceptive units in glabrous skin," J. Physiol, p. pp. 283-300, 1979

[2] Johnson K. O., "The roles and functions of cutaneous mechanoreceptors," in Current Opinion in Neurobiology, Elsevier Science Ltd., 2001, p. $455-461$.

[3] Katz D., "The world of touch," L. Erlbaum, 1925 - 1989

[4] Bueno MA, et al, "COSTaM: Tool Design for a Controlled Tactile Stimulation," AMSE JOURNALS-AMSE IFRATH Publication -2014Modelling C; Vol. 75; $\mathrm{N}^{\circ}$ 2; pp 31-42, 2014.

[5] Massi, F., Vittecoq, E., Chatelet, E., Saulot, A., Berthier, Y., "Design of a tribometer for investigating tactile perception," Proceedings of the Institution of Mechanical Engineers, Part J: Journal of Engineering Tribology, 232(6), 773-784, 2018.

[6] Fagiani R., Massi F., Chatelet E., Berthier Y., Akay A., "Tactile perception by fiction induced vibrations," Tribology International, Vol. 44, Issue 10, Pages 1100-1110, 2011.

[7] Fagiani R., Massi F., Chatelet E., Costes J.P., Berthier Y., "Contact of a Finger on Rigid Surfaces and Textiles: Friction Coefficient and Induced Vibrations," Tribology Letters, Volume 48, Issue 2, pp 145-158, 2012. DOI: 10.1007/s11249-012-0010-0. ISSN: 1023-8883

[8] Camillieri B., Bueno M.A., Fabre M., Juan B., Lemaire-Semail B., Mouchnino L., "From finger friction and induced vibrations to brain activation: Tactile comparison between real and virtual textile fabrics," Tribology International,Vol. 126, 2018, pp 283-296.

[9] Dacleu Ndengue J., et al, "Tactile perception and friction induced vibrations: discrimination of similarly patterned wood-like surfaces," IEEE Transactions on Haptics, Vol. 10(3):409-417., 2017.

[10] Cesini, I., Ndengue, J.D., Chatelet, E., Faucheu, J., Massi, F., "Correlation between friction-induced vibrations and tactile perception during exploration tasks of isotropic and periodic textures," Tribology International, vol. 120, pp. 330-339, 2018. 